\title{
Challenges of Digital Commons: A Qualitative Study of an Automated Dispensing Cabinet in a Paediatric Intensive Care Unit
}

\author{
Valentina LICHTNER ${ }^{\mathrm{a}, \mathrm{b}, 1}$, Mirela PRGOMET ${ }^{\mathrm{b}}$, Peter GATES $^{\mathrm{b}}$, \\ Bryony Dean FRANKLIN ${ }^{\mathrm{a}, \mathrm{c}}$ and Johanna I WESTBROOK ${ }^{\mathrm{b}}$ \\ ${ }^{a}$ Department of Practice and Policy, UCL School of Pharmacy, United Kingdom \\ ${ }^{\mathrm{b}}$ Centre for Health Systems and Safety Research, AIHI, \\ Macquarie University, Australia \\ ${ }^{\mathrm{c}}$ Centre for Medication Safety and Service Quality, Pharmacy Department, \\ Imperial College Healthcare NHS Trust, United Kingdom
}

\begin{abstract}
Missed medication doses can affect patient safety in hospitals. The use of automated dispensing cabinets (ADC) in clinical areas may reduce the occurrence of medications being unavailable at the time of need by alerting pharmacy to replenish stock. However, workarounds in ADC use can affect this capability. We studied nurses' use of an ADC in a paediatric intensive care unit with $\sim 54$ hours of observation, semi-structure interviews (19 nurses; 4 pharmacy/management staff) and review of reported incidents. We found running out of medication a recurrent problem despite the ADC. Contributing factors affecting data entry in the ADC, and therefore replenishment alerts to pharmacy, included: the organisation of medication activities in the ward, nurses' information needs, patients' medication needs, and $\mathrm{ADC}$ design. Running out of medications was a 'tragedy of the commons'. Effective solutions may require going beyond user training and directives for accurate data entry, to work process redesign.
\end{abstract}

Keywords. Medication systems, automation, patient safety, systems thinking

\section{Introduction}

Missed medication doses can affect the safety of hospital inpatients [1]. One reason for missed doses is medication being unavailable on the ward at the time of need [1-3]. Introducing automated dispensing cabinets (ADCs) in clinical areas can contribute to fewer missed medication doses $[4,5]$ through a seemingly simple mechanism: clinicians (usually nurses) removing a medication from the ADC enter data about the amount removed; the ADC automatically calculates the remaining level of stock and alerts the hospital pharmacy when a set threshold is reached; pharmacy can then replenish ward stock ('imprest') in the ADC before the stock runs out. However, use of the ADC in a manner that was not as designers intended (e.g. workarounds), may affect this

\footnotetext{
${ }^{1}$ Corresponding Author, Valentina Lichtner, UCL School of Pharmacy, BMA House, Tavistock Square, London WC1H 9JP, United Kingdom.
} 
mechanism and ADC's contributions to improving stock management and timely medication administration $[6,7]$. This paper reports qualitative findings from a study of an ADC in a paediatric intensive care unit (PICU) with a focus on understanding the contextual reasons for workarounds or patterns of use that may lead to the ward running out of stock at the time of patient need. We then reflect on the implications for how to address such workarounds.

\section{Methods}

The study took place in the PICU of a children's hospital in Sydney (NSW, Australia). The 25 -bed unit provides care to 1400 children a year. At any one time, each patient was assisted by at least two nurses - a nurse always at bedside, and a nurse assisting with retrieving necessary supplies, such as medications stored in the medication room. The hospital implemented an ADC (Omnicell - www.omnicell.com.au) in the PICU to store and dispense medications. The ADC replaced a medication cupboard and fridge located close to the nursing station.

Data collection took place approximately three months post ADC implementation. Qualitative data were collected with observations, semi-structured interviews, analysis of hospital documents, and analysis of hospital patient safety incidents reports.

Observations took place in the medication room, as nurses and pharmacy staff carried out medication activities, including interactions with the ADC. We distributed the hours of observations across different shifts to cover different nurses and pharmacists. We carried out $\sim 54$ hours of observations over 14 days (between 6 June - 24 July 2019). We also observed three, one-to-one ADC 'super user' training sessions with nurses.

We conducted semi-structured interviews with 19 PICU nurses. All nurses present on the ward, during two two-hour 'drop-in' interview sessions we held, were invited to participate. We also sought the views of pharmacy staff, implementation team and hospital management. Sampling of participants was purposive, targeting specific roles responsible for managing imprest medications. Interviews were carried out with pharmacy ADC back-office staff, the ADC trainer, the clinical program director and the deputy director of pharmacy.

We carried out a retrospective review of patient safety incidents voluntarily reported through the hospital incident reporting system. In total, 171 incidents were reported in PICU in relation to medication during the study period (January - June 2019).

A thematic analysis was carried out of observation field notes and interview transcripts with the assistance of qualitative data analysis software (Nvivo). Themes were generated from the data in relation to the aims of the study and to capture significant aspects of the context. We also carried out a qualitative analysis of all the incidents in the dataset to triangulate data from observations and interviews. While we used an inductive approach for the identification of themes across our analysis, we also sought explanations by drawing on a range of theories. 'Running out of medication' emerged as a recurrent theme, which we explained by drawing on the theory of the commons [8].

The project received ethical approval from the Hospitals Research Ethics Committee (Ref. 2018/ETH00716). Incident data were de-identified prior to analysis. Observation sessions and interviews were anonymised at the point of data collection. With consent, interviews were audio-recorded and transcribed, or concurrent field notes were taken. 


\section{Results}

\subsection{Running Out of Medication: A Recurring Problem}

The ADC enabled the hospital pharmacy to remotely monitor the level of imprest medications available in the cabinet, so that stock could be replenished before it ran out. This should have prevented medications not being available in PICU at a time of need. Instead, we found that running out of medication was a recurrent problem experienced in PICU, especially for oral liquid medications (in bottles) kept in the ADC fridge. Throughout our interviews with the nurses we were repeatedly told about this issue, in one case also reported as an incident:

\section{... like clonidine, we always run out of clonidine ... (id23)}

Incident report id109: Nil naloxone in ADC, when called pharmacy they told us to use $A D C$. Double checked ADC nil stock yet pharmacy had said they had not been alerted like they should when stock low/out stock by the ADC system.

The experience of 'running out of medication' came as a surprise to nurses as it contradicted the ADC interface, which showed the medication being available. Nurses reported looking around the ward to see if the medication missing from the ADC was in use with other patients. This process was time consuming and did not necessarily resolve in nurses finding the medication.

\subsection{The Problem of Data Entry and Underlying Contributing Factors}

Nurses were told to 'tell the ADC' the quantity of each medication they removed or returned to the ADC, for the ADC 'to know' the current level of stock. We found that for a variety of reasons accurate data entry of quantities was challenging for nurses. Some medications were used frequently; patients were small children and the doses were often micro-doses; for some medications the dose was not known until it was given; nurses would prepare the dose at the bedside; PICU bays accommodated up to four patients and nurses - each caring for a different patient - shared the space and some of the facilities. When a nurse went to the medication room to take a medication for the patient, the nurse did not have to know the exact dose needed - only that there was sufficient volume (or sufficient medication) in the bottle for the dose to be prepared at the bedside. While the nurse walked to the medication room, other nurses asked them to get a medication for their patients - for this too, the requesting nurses did not need to communicate the exact dose. Back at the bedside with the medication while the nurse prepared the dose for their patient, other nurses also asked to use the medication for their patients. Thus, when a nurse entered into the ADC the exact dose (if known), this may not have been the quantity actually used from that bottle, as other nurses may have used it too. In order to enter the data about the dose in the ADC, nurses sometimes checked the patient electronic medication record on the computer in the medication room. However, this was often found to be too slow for their needs - 'by the time you login and check, it takes a couple of minutes' (id13) and was only usually done for controlled medications.

When nurses realised that stock levels of a medication in the ADC were not accurate, updating the amounts was not straightforward. For fridge items we were told the ADC did not provide 'a bin level' to update - 'when you get out a fridge item there's no-you 
can't say, ooh, zero left, so I took the last bottle. You can't do that' (id16). Thus, updating stock levels seemed to require a laborious process of returning the bottle of medication under a patient name and then removing it again under another patient name - just to correct the quantity - then returning it again, possibly multiple times for all the patients that bottle had been used for.

.... And I put two mls for my patients and then someone goes, 'oh, have you got clonidine, excellent, great; can I just have that before you go back?' And then you forget, oh, hang on, then [...] [I have to] return it for me, take it out for that person, try to remember their dose and how many mls that is, and then return it for that person while I'm there. So it becomes laborious [...] to do it properly.... (id13)

An additional reason for data about use of liquids not being accurate was also syringes' 'dead space' (unaccounted space around the needle $-\sim 0.2 \mathrm{ml}$ liquid extra to the intended dose). As a pharmacist explained, while nurses may enter in the ADC 'the small dose you need', volume may be lost 'when you draw it with the syringe' (field notes, id0708). This small loss accumulates across doses from each bottle over what has been documented in the ADC.

\subsection{Not One Person in Charge of ADC 'Housekeeping'}

After the ADC implementation, pharmacists relied on ADC reports to monitor imprest levels from a distance. They tidied up ADC medication drawers when restocking. Among nurses there appeared to be some confusion about who should be responsible for cleaning the fridge of unnecessary items and checking accuracy of stock levels. It was up to anyone removing/returning medications to pay attention to the stock and take the initiative, but unless nurse were seeking a patient's medication, 'you wouldn't even go' to the ADC.

\section{...If you're not wanting to remove anything you wouldn't even go [to the ADC] (id17}

\subsection{Blaming Nurses' Lack of Compliance}

We sensed among the implementation team a perception that the problem of running out of medications lay with nurses' unwillingness to comply with data entry rules. Similarly, nurses blamed themselves for the problem. They told us that nurses 'don't put what they're taking properly' (id7). As a result, the solution was sought in training and repeated calls for compliance - 'we have to [...] reiterate the importance of writing the right things down' (id3). However, these interventions did not seem to address the issues.

\section{Discussion and Conclusion}

PICU nurses reported an ongoing struggle with keeping accurate accounts of medications in the ADC, resulting in running out of stock. While the directive of completing accurate data entry in the ADC was frequently reinforced, nurses reported they were often unable to comply, or their data entry efforts were insufficient. This problem was especially apparent with medication kept in the fridge. 
We propose that nurses encountered with medications in the ADC fridge the 'tragedy of the commons' [8] similarly to farmers of common pastures finding their resources depleted by the accumulation of individual use. The physical 'common' of the medication bottles in the fridge had a corresponding 'common' in the digital counts recorded in the ADC - the ADC fridge was therefore both a physical and a digital common. Accurate or inaccurate counts depended on the accumulation of individual (data entry) transactions, in parallel to an accumulation of individual (physical) transactions with medications in the ADC fridge (i.e. never remove or return a medication without also 'telling' the ADC, contributing to collective digital counts). The expression 'digital common' usually refers to repositories of informational resources, such as wikis or open-source software [9]. The Internet itself has been considered a commons [10] and the challenges of its development akin to the tragedies of the commons. While the latter are purely digital, the automated dispensing cabinet we studied reflected also a 'physical reality' of medication stock.

Unless work processes are changed (e.g. all doses are prepared in the medication room), training and calls for compliance are likely to have only limited effect in resolving ADC issues of this kind. Instead, it is necessary to understand nurses' information needs, clinical needs of their patients, and context-specific medication work practices, as these contribute to data entry issues and therefore 'tragedies of the commons' with the ADC. Nominating a person per shift responsible for keeping an eye on the commons may, for example, be a more effective solution to the problem of running out of medications than telling nurses to do what they cannot do.

\section{Acknowledgements}

This project has received funding from the European Union's Horizon 2020 research and innovation programme under the Marie Sklodowska-Curie grant agreement No 740131 and NHMRC Partnership Projects Grant APP1094878.

\section{References}

[1] Rostami P, Heal C, Harrison A, Parry G, Ashcroft DM, Tully MP. Prevalence, nature and risk factors for medication administration omissions in English NHS hospital inpatients: a retrospective multicentre study using Medication Safety Thermometer data. BMJ open 9 (2019), e028170.

[2] Morley C, McLeod E, McKenzie D et al. Reducing dose omission of prescribed medications in the hospital setting: a narrative review. Drugs \& Therapy Perspectives 32 (2016), 203-208.

[3] Hunt KV, Harding AM, Taylor SE, Curtain C. Evaluation of medication dose omissions amongst inpatients in a hospital using an electronic Medication Management System. Journal of evaluation in clinical practice 24 (2018), 688-694.

[4] Boyle CM, Maxwell DJ, Meguid SE, Ouaida D, Krass I. Missed doses: An evaluation in two drug distribution systems. The Australian Journal of Hospital Pharmacy 28 (1998), 413-416.

[5] Schwarz HO, Brodowy BA. Implementation and evaluation of an automated dispensing system. Am J Health Syst Pharm 52 (1995), 823-828.

[6] Koopman P, Hoffman RR. Work-arounds, make-work, and kludges. IEEE Intelligent Systems 18 (2003), 70-75.

[7] Azad B, King N. Enacting computer workaround practices within a medication dispensing system. European Journal of Information Systems 17 (2008), 264-278.

[8] Hardin G. The tragedy of the commons. Science 162 (1968), 1243-1248.

[9] Digital commons. In, Wikipedia, 2019.

[10] V.G. Cerf, Revisiting the tragedy of the commons. Commun. ACM 56 (2013), 7-7. 